\title{
Sistematização da assistência de enfermagem à criança com sífilis congênita: relato de experiência
}

\author{
Systematization of nursing care for children with congenital syphilis: experience report
}

Sistematización de la atención de enfermaría al niño con sífilis congénita: informe de experiencia

João Matheus Ferreira do Nascimento ${ }^{1 *}$, Ivanildo Gonçalves Costa Júnior ${ }^{1}$, Maria Clara Feijó de Figueiredo ${ }^{1}$, Danila Barros Bezerra Leal ${ }^{2}$, Francisco João de Carvalho Neto², Michelly Moura Feijó $^{3}$, Michelle Marinho Ramos ${ }^{4}$, Rômulo Rufino Alves Figueiredo ${ }^{5}$, Cinara Maria Feitosa Beleza², Denival Nascimento Vieira Júnior ${ }^{6}$.

\section{RESUMO}

Objetivo: Relatar a experiência de acadêmicos de enfermagem durante a aplicação do processo de enfermagem a um recém-nascido com sífilis congênita. Relato de experiência: As aulas práticas hospitalares aconteceram em um serviço de referência de uma cidade da macrorregião no Centro-Sul piauiense, no segundo semestre de 2019. Dentre as atividades desenvolvidas na disciplina destaca-se o acompanhamento detalhado de um caso, de acordo com a sistematização da assistência de enfermagem. Foi implementado o processo de enfermagem, seguindo as cinco etapas, fazendo uso das taxonomias NANDA, NIC e NOC. O caso relatado trata-se da condição de Sífilis Congênita diagnosticada em uma recém-nascido, foram realizadas três visitas, para identificação, implementação, acompanhamento e avaliação dos cuidados de enfermagem. Considerações finais: A sistematização da assistência de enfermagem contribui de forma considerável na qualidade da assistência, permitindo a implementação e avaliação de intervenções nos indivíduos de forma integral. Os acadêmicos vivenciaram a aplicação do processo de enfermagem em indivíduos em situação de hospitalização, essas experiências contribuem diretamente na qualidade da formação desses profissionais.

Palavras-chave: Processo de enfermagem, Sífilis congênita, Assistência de enfermagem, Sistematização da assistência de enfermagem, Relatos de casos.

\section{ABSTRACT}

Objective: To report the experience of nursing students during the application of the nursing process to a newborn with congenital syphilis. Experience report: The practical hospital classes took place in the reference service in a city in the macro-region in the Center-South of Piauí, in the second half of 2019. Among the activities developed in the discipline, the detailed monitoring of a case, according to the systematization of nursing care, stands out. The nursing process was implemented, following the five steps, using the NANDA, NIC and NOC taxonomies. The case reported is about the condition of Congenital Syphilis diagnosed in a newborn, three visits were made, for identification, implementation, monitoring and evaluation of nursing care. Final considerations: The systematization of nursing care contributes considerably to the quality of care, allowing the implementation and evaluation of interventions in individuals in a comprehensive way. The students experienced the application of the nursing process to individuals in a hospitalization situation, these experiences directly contribute to the quality of these professionals' education.

Keywords: Nursing process, Syfilis congenital, Nursing care, Systematization of nursing care, Cases reports.

\section{RESUMEN}

Objetivo: Relatar la experiencia de estudiantes de enfermería durante la aplicación del proceso de enfermería a un recién nacido con sífilis congénita. Informe de experiencia: Las clases prácticas hospitalarias se llevaron a cabo en el servicio de referencia en una ciudad de la macrorregión del Centro-Sur de Piauí, en el segundo semestre de 2019. Entre las actividades desarrolladas en la disciplina, se destaca el seguimiento detallado de un caso, según el sistematización del cuidado en enfermería. El proceso de enfermería se implementó, siguiendo los cinco pasos, utilizando las taxonomías NANDA, NIC y NOC. El caso reportado es sobre la condición de Sífilis Congénita diagnosticada en un recién nacido, se

\footnotetext{
${ }^{1}$ Universidade Federal do Piauí (UFPI), Picos - PI. E-mail: matheus_fn12@hotmail.com

2 Universidade Federal do Piauí (UFPI), Teresina - PI.

${ }^{3}$ Universidade de Pernambuco (UPE), Recife - PE.

${ }^{4}$ Faculdade Adelmar Rosado (FAR), Teresina - PI.

${ }^{5}$ Universidade Federal do Pernambuco (UFPE), Recife - PE.

${ }^{6}$ Universidade de São Paulo (USP), São Paulo - SP.
} 
realizaron tres visitas, para la identificación, implementación, seguimiento y evaluación de los cuidados de enfermería. Consideraciones finales: La sistematización de los cuidados de enfermería contribuye considerablemente a la calidad de los cuidados, permitiendo la implementación y evaluación de las intervenciones en los individuos de manera integral. Los estudiantes vivieron la aplicación del proceso de enfermería a personas en situación de hospitalización, estas experiencias contribuyen directamente a la calidad de la formación de estos profesionales.

Palabras clave: Proceso de enfermería, Sífilis congénita, Atención de enfermería, Sistematización de la atención de enfermería, Informes de casos.

\section{INTRODUÇÃO}

A sífilis é um problema de saúde pública a nível mundial, especificamente a Sífilis Congênita (SC), evidenciada pela transmissão hematogênica do Treponema pallidum, da gestante infectada inadequadamente tratada para o seu concepto, por via transplacentária (MINISTÉRIO DA SAÚDE, 2018; BRANCO TJT, et al., 2020).

A partir do ano de 2005 entraram para a Lista de Agravos e Notificação Compulsória como uma estratégia de controle da transmissão vertical da doença. No entanto, em 2011 a Organização Mundial da Saúde (OMS) estimou que em todo mundo, anualmente ocorram 12 milhões de casos de sífilis, sendo $90 \%$ em países em desenvolvimento, o que evidencia um grave problema de saúde pública (MAGALHÃES DMS, et al., 2011; SILVA LMC, et al., 2019).

De acordo com dados do Departamento de Informática do Sistema Único de Saúde (DATASUS), casos de SC em menores de um ano de idade, em 2018 foi de 26.219. A idade materna mais comum é entre 20 a 29 anos com 54,7\% (DATASUS, 2019). Existem outros fatores que preocupam para a incidência desse agravo, dentre eles: cor, raça e grau de escolaridade das mães e também a vulnerabilidade social (SILVA DAR, et al., 2017).

A principal forma de prevenção seria um pré-natal e puerpério apropriados, unidos de um acompanhamento continuado dos serviços de saúde em meio ambulatorial ou domiciliar, sendo elencada a participação do parceiro para um bom acolhimento, orientações, rastreamento e participação da família no tratamento, fortalecendo o vínculo afetivo para efetivação da promoção em saúde contra a doença (LAZARINI FM e BARBOSA DA, 2017).

Além disso, outro aspecto importante no acompanhamento para efetivação do cuidado é a aplicação de métodos sistematizados, fomentado sob a Sistematização da Assistência de Enfermagem, bem como o Processo de Enfermagem, pois auxilia na organização das etapas do cuidado (GARCIA TR, 2016).

Nesse contexto, elencou-se a seguinte questão norteadora: "Qual a influência da sistematização de enfermagem na atenção aos cuidados de um recém-nascido com sífilis congênita em situação de vulnerabilidade social?". Justificando a realização deste estudo de caso pela importância em se abordar de maneira sistematizada um problema de saúde pública associada a fatores de susceptibilidade ambiental e social, como a SC. O objetivo foi relatar a experiência de acadêmicos de enfermagem durante a aplicação da sistematização da assistência de enfermagem à um recém-nascido com SC.

\section{RELATO DE EXPERIÊNCIA}

Trata-se de um relato de experiência, de graduandos enfermagem ao acompanhamento de um recémnascido com sífilis congênita. A experiência iniciou durante as práticas do estágio em saúde da criança e do adolescente, sendo realizada três consultas de enfermagem.

A experiência das consultas possibilitou a realização das avaliações completas: histórico familiar; análise socioeconômica e de situações de risco e vulnerabilidade; anamnese e exame físico, verificando a presença dos reflexos subcorticais e marcos de desenvolvimento; registro das evoluções; prescrição dos diagnósticos de enfermagem NANDA, implementação de intervenções NIC e Resultados esperados NOC. Orientamos os familiares quanto as vacinações, higiene, nutrição e marcos de desenvolvimento. Assim, construído o genograma familiar. 


\section{Histórico de Enfermagem}

Foi realizada a coleta de informações do histórico para identificar evidências e fatores de riscos associados a origem ou decurso da doença. O RN termo, sexo masculino, negro, se demonstrou ativo, normocorado e com padrão de sono normal. A criança apresentava vestimentas adequadas e bom padrão higiênico, o mesmo é banhado uma vez por dia, tendo as eliminações vesicais e intestinais presentes. Foi vacinado com as vacinas BCG e Hepatite $B$, além de estar sob tratamento para sífilis congênita com penicilina uma vez ao dia, quando realizado o exame Veneral Disease Research Laboratory (VDRL), o resultado foi soro reagente.

O nascimento por parto vaginal, no Hospital de referência da região, com 38 semanas de idade gestacional e o parto não houve intercorrências. O peso ao nascer foi de 3.290 gramas, 49 centímetros de comprimento, perímetro cefálico de 35 centímetros, perímetro torácico de 33 centímetros e perímetro abdominal com 33 centímetros. Quando realizado o Índice de Apgar no $1^{\circ}$ minuto de vida, o escore foi 8 , e no $5^{\circ}$ minuto de vida, foi 9. O RN seguia em aleitamento materno exclusivo e ainda não havia sido realizado o teste do pezinho. A mãe possui 29 anos, ensino fundamental I incompleto e trabalha como lavadeira de roupas. Quando perguntada sobre o histórico gestacional, esta foi a sua sexta gestação, com três nascidos vivos e todas não planejadas, porém, demonstrou aceitação.

A realização das consultas do Pré-natal foi incompleta, feita apenas duas consultas. Ao realizar exames solicitados, foi diagnosticada com sífilis, na qual perpetuou para suas duas últimas gestações, no entanto, seu conjugue não possui a infecção. Quando investigada sobre a situação ambiental e residencial, a residência possui água encanada e tratada, energia elétrica e coleta de lixo, contudo é evidente a vulnerabilidade social.

\section{Diagnósticos de Enfermagem}

O diagnóstico de enfermagem é subsidiado pela taxonomia NANDA, no qual dispõe da apreciação e julgamento de casos da condição de saúde e processo de vida e/ou vulnerabilidade do indivíduo, família ou grupo, possibilitando prever, detectar e controlar potenciais complicações, facultando a sistematização da assistência e do serviço, bem como a sua continuidade (MENDES FA, et al., 2018).

Quanto aos diagnósticos em estudo, tanto reais quanto de risco, foram buscados destacar seu título das classes a partir de um domínio, também seus fatores relacionados e características definidores que mostram os conceitos de tais termos (Quadro 1).

Quadro 1 - Diagnóstico de Enfermagem Baseado na Taxonomia NANDA.

\begin{tabular}{|c|c|}
\hline & OMIA NA \\
\hline Domínio/Classe & iagnóstico \\
\hline Promoção/Controle da saúde & $\begin{array}{l}\text { Disposição para controle aumentando do regime terapêutico } \\
\text { caracterizado pela pouca dificuldade com o regime de tratamento } \\
\text { prescrito e não haver aceleração inesperada dos sintomas da doença. }\end{array}$ \\
\hline Promoção/Controle d & $\begin{array}{l}\text { Manutenção do lar prejudicada relacionada ao conhecimento deficiente, } \\
\text { a doença, com finanças insuficientes e planejamento familiar insuficiente, } \\
\text { evidenciado por ambiente desordenado, membros da família } \\
\text { sobrecarregados e dificuldade em manter a casa confortável. }\end{array}$ \\
\hline $\begin{array}{l}\text { Enfrentamento/tolerância } \\
\text { estresse }\end{array}$ & $\begin{array}{l}\text { Risco de comportamento desorganizado do bebê evidenciado por } \\
\text { problemas orais, procedimento dolorosos e invasivos. }\end{array}$ \\
\hline Segurança/proteção & $\begin{array}{l}\text { Mucosa oral prejudicada relacionado a infecção e comprometimento } \\
\text { imunológico, evidenciada por fissuras, vesículas, placas } \\
\text { esbranquiçadas, nódulos e manchas. }\end{array}$ \\
\hline Confo & $\begin{array}{l}\text { aracterizado por efeitos } \\
\text { mentoso, estímulos am } \\
\text { abilidade e recursos de }\end{array}$ \\
\hline
\end{tabular}

Fonte: Nascimento JMF, et al., 2021; dados baseados em: Herdman TH, 2018.

\section{Intervenções}

As intervenções de Enfermagem baseiam-se no desempenho das ações sobre o paciente, isto é, executar as atividades de cuidado prescritas pela taxonomia NIC, como demonstrado no Quadro 2, a fim de padronizar a descrição dos cuidados interventivos para o paciente, moldadas segundo a presença da enfermagem nos casos, para que os resultados esperados sejam alcançados (DOCHTERMAN JM e BULECHEK GM, 2016). 
Quadro 2 - Intervenções de Enfermagem baseado na taxonomia NIC.

\begin{tabular}{|l|l|}
\hline \multicolumn{2}{|c|}{ TAXONOMIA NIC } \\
\hline Domínio/Classe & Diagnóstico \\
\hline $\begin{array}{l}\text { Disposição } \\
\text { autocontrole da saúde } \\
\text { melhorado }\end{array}$ & $\begin{array}{l}\text { Avaliação da saúde; educação em saúde; facilitação da aprendizagem; } \\
\text { identificação de risco; facilitação da autorresponsabilidade; melhora do } \\
\text { sistema de apoio; promoção do envolvimento familiar. }\end{array}$ \\
\hline $\begin{array}{l}\text { Manutenção do lar } \\
\text { prejudicada }\end{array}$ & $\begin{array}{l}\text { Apoio familiar; assistência ao autocuidado em atividade essenciais da vida } \\
\text { diária; melhora do papel; promoção da paternidade/maternidade; } \\
\text { aconselhamento; apoio ao cuidador; controle do ambiente e preparo do lar; } \\
\text { ensino ao indivíduo. }\end{array}$ \\
\hline $\begin{array}{l}\text { Risco de comportamento } \\
\text { desorganizado do lactente }\end{array}$ & $\begin{array}{l}\text { Aconselhamento para lactação; controle da dor; controle do ambiente e } \\
\text { conforto; cuidados com o recém-nascido; identificação de risco; } \\
\text { monitorização de sinais vitais; monitorização nutricional; sucção não nutritiva; } \\
\text { supervisão. }\end{array}$ \\
\hline Mucosa oral prejudicada & $\begin{array}{l}\text { Assistência no autocuidado; controle da nutrição; controle hídrico; } \\
\text { manutenção da saúde oral; controle da dor; controle de medicamentos; } \\
\text { cuidados com lesões. }\end{array}$ \\
\hline Conforto prejudicado & $\begin{array}{l}\text { Aumento da segurança; banho; controle da dor; controle do ambiente e } \\
\text { conforto; melhora do sistema de apoio; técnica para acalmar; controle de } \\
\text { medicamentos; melhora do sono. }\end{array}$ \\
\hline
\end{tabular}

Fonte: Nascimento JMF, et al., 2021; dados baseados em: Dochterman JM e Bulechek GM, 2016.

\section{Avaliação do Paciente}

O planejamento do cuidado é instituído após a determinação dos diagnósticos de enfermagem (NANDA), no qual, são traçados os objetivos do cuidado, que serão as metas a serem atingidas com as intervenções, isto é, os resultados esperados para cada diagnóstico de enfermagem definido (MOORHEAD S, et al., 2016). Para isso, utiliza-se a taxonomia padronizada NOC (Quadro 3).

Quadro 3 - Objetivos de Enfermagem Baseado na Taxonomia NOC.

\begin{tabular}{|c|c|}
\hline & IOC \\
\hline Domínio/Classe & Diagnóstico \\
\hline $\begin{array}{l}\text { Disposição para } \\
\text { autocontrole da saúde } \\
\text { melhorado }\end{array}$ & $\begin{array}{l}\text { Autocontrole da medicação; comportamento de aceitação do medicamento } \\
\text { prescrito; conhecimento de controle da infecção; conhecimento do } \\
\text { funcionamento sexual na gravidez e no pós-parto; conhecimento dos } \\
\text { procedimentos de tratamento e regime de tratamento; controle de riscos do } \\
\text { processo infeccioso; controle dos sintomas; participação nas decisões sobre } \\
\text { cuidados de saúde. }\end{array}$ \\
\hline $\begin{array}{l}\text { Manutenção do lar } \\
\text { prejudicada }\end{array}$ & $\begin{array}{l}\text { Ambiente domiciliar seguro; bem-estar familiar; desempenho de papel; estado de } \\
\text { conforto e ambiente; apoio social; autocontrole da doença; conhecimento dos } \\
\text { recursos de saúde; funcionamento familiar; saúde emocional do cuidador. }\end{array}$ \\
\hline $\begin{array}{ll}\text { Risco } & \text { de } \\
\text { comportamento } & \\
\text { desorganizado } & \text { do } \\
\text { lactente } & \\
\end{array}$ & $\begin{array}{l}\text { Adaptação do recém-nascido; conhecimento da criação de filhos e dos cuidados } \\
\text { com o lactente; controle de riscos; desenvolvimento da criança; detecção do } \\
\text { risco; estado de conforto ambiental; saúde oral; sono; termorregulação do recém- } \\
\text { nascido. }\end{array}$ \\
\hline $\begin{array}{l}\text { Mucosa } \\
\text { prejudicada }\end{array}$ & $\begin{array}{l}\text { Integridade tissular da pele e mucosas; saúde; estado de deglutição na fase oral; } \\
\text { nível de dor; cuidado com a higiene oral; conhecimento de controle da infecção; } \\
\text { conhecimento da promoção em saúde; controle de riscos do processo infeccioso; } \\
\text { estado imunológico; estado nutricional ingestão de alimentos e líquidos; resposta } \\
\text { ao medicamento. }\end{array}$ \\
\hline Conforto prejudicado & $\begin{array}{l}\text { Estado de conforto, físico, ambiental, psicoespiritual e sociocultural; controle de } \\
\text { sintomas; efeitos nocivos da dor; sono; adaptação a mudança ambiente domiciliar } \\
\text { seguro; apoio social; resposta ao medicamento. }\end{array}$ \\
\hline
\end{tabular}

Fonte: Nascimento JMF, et al., 2021; dados baseados em: Moorhead S, et al., 2016.

\section{Evolução de Enfermagem}

Na primeira visita, realizada em 17 de setembro de 2019, por volta das 16:30 horas, realizou-se o exame físico completo, destacando-se os principais pontos. O Recém-nascido com 9 dias de vida, se mostrou ativo, com membros fletidos, normocorado, com presença de lanugem. Observou-se que a cabeça possuía fontanelas planas e normotensas, a mucosa ocular estava normocorada, as pupilas isocóricas e 
fotorreagentes, sem apresentação de edemas e secreções. O pavilhão auricular estava adequado e sem alterações, a mucosa oral com presença de placas amareladas, o palato integro, as narinas sem desvio de septo. As mamas sem edemas e sem secreções.

Ao exame respiratório, o $\mathrm{RN}$ estava eupneico, com sons respiratórios normais. A avaliação cardiovascular se mostrou normal e sem sopro. Acerca da inspeção da pele, as extremidades estavam aquecidas, os pulsos periféricos presentes, o abdome globoso, o coto mumificado e ruídos hidroaéreos presentes. À inspeção genital, os testículos locados, o pênis e uretra sem alterações visíveis. Todos os reflexos possíveis de serem testados estavam presentes. Assim, o recém-nascido seguiu em Aleitamento Materno Exclusivo (AME).

A Mãe foi orientada sobre AME, realizou-se a limpeza do coto umbilical e sífilis. Realizada a antropometria do RN, Comprimento geral: $49 \mathrm{~cm}$, perímetro cefálico $35 \mathrm{~cm}$, perímetro torácico $33 \mathrm{~cm}$ e perímetro abdominal $33 \mathrm{~cm}$. Verificados os sinais vitais, a Frequência Cardíaca: 145 batimentos por minuto, e a Frequência Respiratória: 51 respirações por minuto.

A Segunda Visita: realizada em 15 de outubro de 2019 as 17:50 horas. O RN se encontrava em domicílio. Realizou-se o exame físico completo, sendo os principais dados: estado geral ótimo, sem alterações na pele, expressões ou movimentos, apresenta-se normocefálico, fontanelas planas e firmes, sem edemas ou massas. As pupilas estavam isocóricas e fotorreagentes. Orelha integra, acuidade auditiva preservada. O nariz sem alterações. Lábios íntegros e normocorados, mucosa oral preservada. O pescoço não apresentava gânglios edemaciados, nem massas ou desvio de traqueia.

A Inspeção estática não apontou alterações na forma do tórax. Padrão respiratório eupneico. Durante a ausculta, foram obtidos sons do tipo claro pulmonar. Na avaliação cardíaca, a inspeção não apontou nenhuma alteração na caixa torácica. $\mathrm{Na}$ ausculta, foram encontrados murmúrios vesiculares em toda sua extensão, sem ruídos adventícios. No abdome, cicatriz umbilical sem alterações e ruídos hidroaéreos presentes. Palpado e sentido os pulsos femorais.

No exame das genitálias foi realizada uma breve anamnese com a mãe, e na inspeção não foi observado nenhuma alteração. Abertura do canal uretral localizado na região central da glande e prepúcio possível de retrair. Feita também a palpação para verificar a locação dos testículos. Observado o orifício anal quanto a sua integridade. Eliminações vesicais e intestinais presentes. Avaliada a pega na mama e reforçado as orientações sobre sífilis. Foi entregue preservativos, assim como, feitas orientação sobre métodos contraceptivos para os pais. A Mãe foi orientada sobre o aleitamento materno exclusivo, higiene íntima e bucal e estimulação do desenvolvimento. Comprimento: $54 \mathrm{~cm}$, perímetro cefálico $37 \mathrm{~cm}$. FC: $106 \mathrm{bpm}$. FR: $36 \mathrm{rpm}$.

A terceira visita, realizada em 18 de novembro de 2019, às 17:00 horas. Na avaliação: o RN estava normocefálico, fontanela posterior fechada. Acuidade auditiva preservada. Mucosa oral preservada. Avaliação pulmonar normal. Padrão respiratório eupneico. Na avaliação cardíaca, não foram observadas nenhuma alteração. Eliminações vesicais e intestinais presentes. Avaliada a pega da criança, reforço sobre a higiene ambiental e pessoal, esclarecimento de dúvidas quanto alimentação e educação sexual para os pais.

Segue em AME, reforçado as orientações sobre higiene íntima e bucal, todos os marcos para sua faixa etária se mostraram presentes e vacinação em dias. Realizado a antropometria e sinais vitais, comprimento: $60 \mathrm{~cm}$, perímetro cefálico $40 \mathrm{~cm}$. frequência cardíaca: 103 batimentos por minuto e frequência respiratória de 35 respirações por minuto.

\section{Genograma}

Após a realização das coletas de dados de enfermagem acerca da situação clínica do recém-nascido, pode-se buscar dados familiares e sociais, a fim de se investigar tais fatores que implicaram na interferência do caso e geração da SC no recém-nascido, podendo gerar a criação de um genograma familiar como ferramenta para entender tais influencias da história familiar na doença estudada.

Para preservar a privacidade da situação familiar, os nomes foram substituídos por numerais, referenciados como: Pessoa 1, 2, 3, 4, 5 e 6. As informações destacadas foram analisadas e ilustradas mediante a apresentação da Figura 1, resultado da montagem do genograma familiar em questão. 
Na história familiar analisada, pessoa 1 teve um relacionamento com a pessoa 2, e desse relacionamento se originou dois filhos do sexo masculino, um deles é a pessoa 3, que se casou com a pessoa 4, filha de pessoa 5 já falecida por câncer de mama, e pessoa 6 , além da pessoa 4 , há outros três filhos entre pessoa 5 e pessoa 6 , sendo dois homens e uma mulher.

A relação entre o casal, pessoa 3 e pessoa 4, é conflituosa e evidenciada por questões de fidelidade, alcoolismo e finanças. Pessoa 3 tem problemas com alcoolismo, assim como o seu pai, pessoa 1. Os conjugues, pessoa 3 e pessoa 4, tem três filhos nascidos-vivos e três abortos espontâneos, dos filhos oriundos do casal todos são do sexo masculino e os dois mais novos nasceram com sífilis congênita, ambos foram tratados e estão bem até então. Pessoa 4 foi diagnosticada com sífilis por duas vezes e não realizou 0 tratamento adequado e, ao realizar os exames, pessoa 3 não foi detectado. Todos as pessoas citadas nesse contexto familiar são carentes e não possuem empregos formais.

Figura 1 - Genograma familiar de identificação dos fatores de vulnerabilidade familiar.

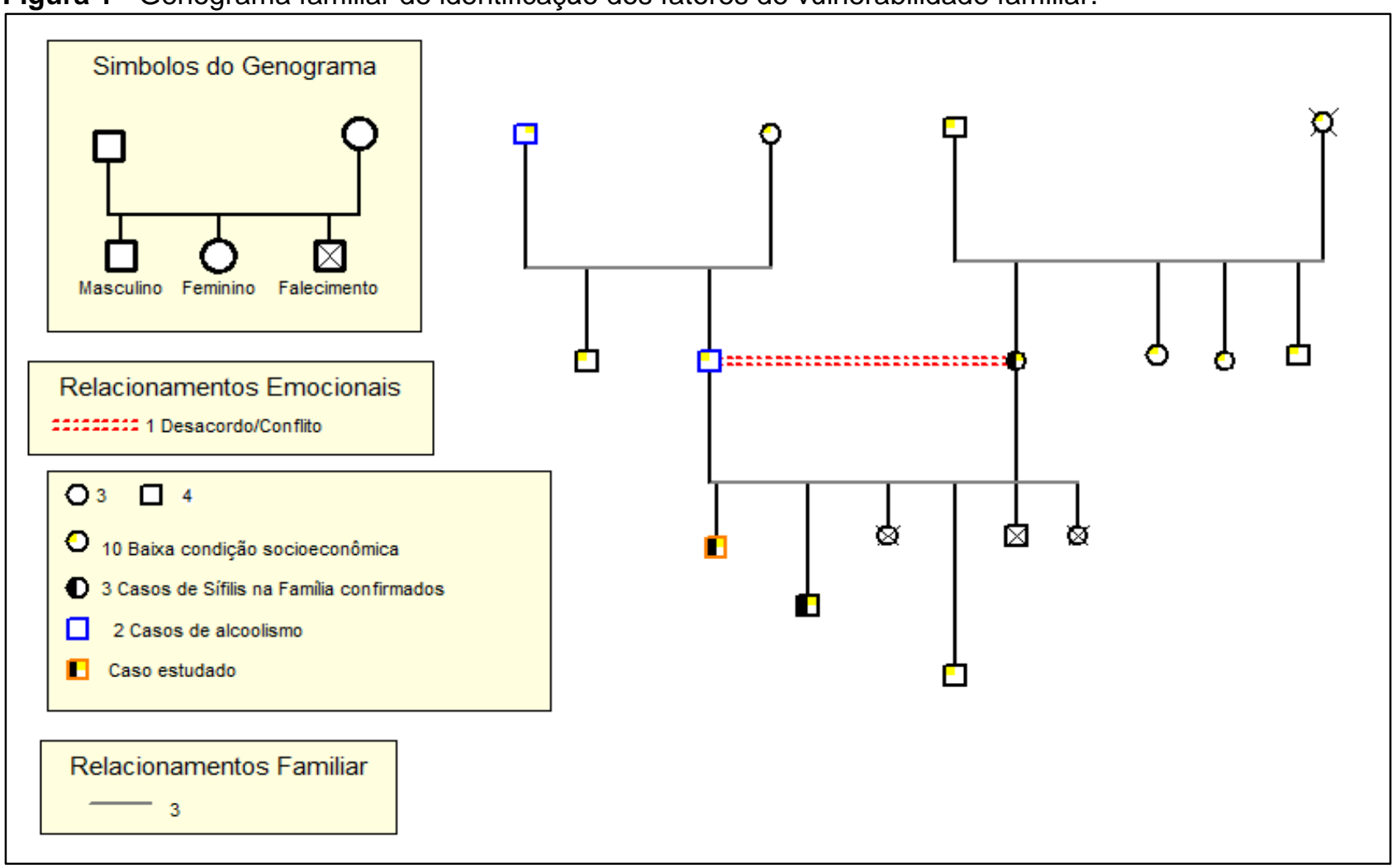

Fonte: Nascimento JMF, et al., 2021; utilizado o programa: GenoPró, 27.

\section{DISCUSSÃO}

O Ministério da Saúde em 2011, como medida de combate à epidemia de SC, tornou competência fundamental das ações de pré-natal na atenção básica, a realização de testes rápidos de sífilis em todas as gestantes e seus parceiros, bem com, da realização do teste na maternidade, para as de sorologia desconhecida ou que não o fizeram durante o pré-natal, a fim de controlar a transmissão vertical da doença, implementar melhores formas de prevenção, diagnóstico precoce e tratamento adequado (SILVA DAR, et al., 2017).

Apesar da eliminação da SC estar pautada em um pré-natal de qualidade, além dos protocolos de diagnóstico durante a preparação do parto, observa-se que, ainda ocorrem casos de SC e sífilis materna, em que a ocorrência pode se relacionar a negligência, seja por falta de planejamento familiar ou desconhecimento (NUNES IR, et al., 2020), soma-se a descoberta tardia pelos serviços de acompanhamento, como demonstra Lafetá KRG, et al. (2016), em um estudo realizado em Minas Gerais, 62,4\% das gestantes investigadas descobriram a enfermidade no momento do parto ou da curetagem. 
Não obstante, a sífilis congênita é uma doença de fácil prevenção, e a redução da transmissão vertical da sífilis faz parte da política de prevenção da mortalidade materno-infantil, preconizada pelo MS mediante a Rede Cegonha, que busca garantir a triagem e acompanhamento durante o pré-natal e pré-parto (COOPER JM e SÁNCHEZ PJ, 2018; BOMFIM VVBS, et al., 2021). Assim, denota-se que uma realização ineficaz do pré-natal para diagnóstico ou número de consultas insuficientes para acompanhamento do tratamento tenha ocorrido, evidenciando principalmente uma falha no desempenho da educação em saúde, que tem como papel fundamental educar a mãe e os familiares para a adoção do tratamento completo, uma vez que, este não é o primeiro caso no núcleo familiar (CAMPOS CO e CAMPOS CO, 2020).

Avalia-se, portanto, que o controle dos casos de SC por meio da detecção precoce é o ideal. Contudo, destaca-se a necessidade do acompanhamento de tais casos mesmo após o tratamento, como prevenção de reincidência, dado pelas ações de educação em saúde, principalmente quanto ao uso de preservativo (SOUSA AT, et al., 2021).

Questões que envolvem o parceiro, tanto em relação ao tratamento, como cuidados para a prevenção de reinfecção são de extrema necessidade. Assim, torna-se importante a avaliação completa de casos suspeitos de SC em famílias com histórico da doença, falta de um pré-natal completo e adequado ou mesmo, com identificação de risco por falta de planejamento familiar (SILVEIRA CR, et al., 2020; SILVA MA, et al., 2021).

Desta maneira, é imprescindível a realização de avaliação por meio de um cuidado sistematizado como é a Sistematização da Assistência de Enfermagem, capaz de identificar falhas decorrentes de uma assistência precária e organizar melhor o protocolo de cuidados a ser seguido, traçando metas e estabelecendo a reavaliação das ações prestadas.

A utilização de diferentes taxonomias é de grande valia para a padronização da linguagem empregada pelos profissionais de enfermagem, recomendando e viabilizando a atuação adequada na execução da assistência e resolução de casos. A realização de um estudo aprofundado sobre uma infecção vertical, com observação aos fatores de risco, expõe a dimensão do desenvolvimento de um processo saúde doença e as implicações em se combater a doença, prevenir à recidiva e a transmissão, isto é, o impacto da prevenção para a saúde de vários indivíduos.

A experiência adquirida é de suma relevância para a formação, tal qual, obteve um conhecimento prático e posteriormente teórico de uma situação de grande incidência, considerado um problema de saúde pública, necessitando que o profissional esteja devidamente capacitado para atender as particularidades de cada caso e as dificuldades de aproximação da família.

\section{REFERÊNCIAS}

1. BOMFIM VVBS, et al. A importância do pré-natal no diagnóstico e tratamento da sífilis congênita. Revista Eletrônica Acervo Saúde, 2021; 13(7): 1-9.

2. BRANCO TJT, et al. Perfil Epidemiológico dos casos notificados de sífilis congênita no estado do Acre nos anos 20092018. Revista Eletrônica Acervo Saúde, 2020; 12(9): 1-10.

3. CAMPOS CO, CAMPOS CO. Abordagem diagnóstica e terapêutica da sífilis gestacional e congênita: revisão narrativa. Revista Eletrônica Acervo Saúde, 2020; 53: e3786.

4. COOPER JM, SÁNCHEZ PJ. Congenital Syphilis. Semin Perinatol. 2018; 42(3): 176-84.

5. DOCHTERMAN JM, BULECHEK GM. Classificação das Intervenções de Enfermagem (NIC). 6ª ed. Porto Alegre: Artmed, 2016.

6. GARCIA TR. Sistematização da Assistência de Enfermagem: Aspecto Substantivo da Prática Profissional. Rev. Esc. Anna Nery, 2016; 20(1): 5-10.

7. HERDMAN TH. Diagnósticos de enfermagem da NANDA: definições e classificação 2018-2020. Porto Alegre: Artmed, 2018.

8. LAFETÁ KRG, et al. Maternal and congenital syphilis, underreported and difficult to control. Rev Bras Epidemiol, 2016; 19(1): 63-74.

9. LAZARINI FM, BARBOSA DA. Intervenção Educacional na Atenção Básica para Prevenção de Sífilis Congênita. Rev. Latino-Am. Enfermagem, 2017; 25(1): 1-9.

10. MAGALHÃES DMS, et al. A Sífilis na Gestação e Sua Influência na Morbimortalidade Materno-infantil. Rev. Com. Ciências Saúde, 2011; 22(1): 43-54. 
11. MENDES FA, et al. Diagnósticos de Enfermagem em portadoras de hipertensão arterial na atenção primária. Rev. Estação Científica UNIFAP, 2018; 8(1): 91-101.

12. MINISTÉRIO DA SAÚDE. Secretaria de Vigilância em Saúde. Cuidado Integral à Pessoa com Sífilis: História Natural da Doença, Prevenção, Diagnóstico e Tratamento. 2018. Disponível em: https://portaldeboaspraticas.iff.fiocruz.br/biblioteca/cuidado-integral-a-pessoa-com-sifilis-historia-natural-da-doencaprevencao-diagnostico-e-tratamento/. Acessado em: 21 de julho de 2021.

13. MINISTÉRIO DA SAÚDE. Sistema de Informações sobre Mortalidade. Disponível em: http://indicadoressifilis.aids.gov.br/. Acessado em: 21 de julho de 2021.

14. MOORHEAD S, et al. Classificação dos Resultados de Enfermagem (NOC): mensuração dos resultados em saúde. $5^{\text {a }}$ ed. Rio de Janeiro: Elsevier, 2016.

15. NUNES IR, et al. Sífilis Congênita: caracterização epidemiológica no estado do Piauí, Brasil. Revista Eletrônica Acervo Saúde, 2020; 1(50): 1-7.

16. SILVA DAR, et al. Prevalência de Sífilis em Mulheres. Rev. Enferm. Foco. 2017; 8(3): 61-64.

17. SILVA LMC, et al. Sífilis congênita no estado do Pará-Brasil, 2007 a 2016. Revista Eletrônica Acervo Saúde, 2019; 24(e1003): 1-10.

18. SILVA MA, et al. A assistência de enfermagem no pré-natal em gestantes diagnosticadas com sífilis: através de uma revisão integrative. Revista Eletrônica Acervo Saúde, 2021; 11(1): 1-9.

19. SILVEIRA CR, et al. Papel do enfermeiro na inserção dos parceiros no pré-natal e tratamento de gestantes com sífilis. Revista Eletrônica Acervo Saúde, 2020; 12(11): 1-12.

20. SOUSA AT, et al. Impactos maternos da Sífilis durante a gestação: uma revisão bibliográfica. Revista Eletrônica Acervo Saúde, 2021; 13(4): 1-7. 